\title{
OBSTACLE PROBLEMS AND MAXIMAL OPERATORS
}

\author{
PABLO BLANC, JUAN P. PINASCO, AND JULIO D. ROSSI
}

\begin{abstract}
Fix two differential operators, $L_{1}$ and $L_{2}$ and define a sequence of functions inductively by considering $u_{1}$ as the solution for the Dirichlet problem for an operator $L_{1}$ and then $u_{n}$ as the solution to the obstacle problem for an operator $L_{i}(i=1,2$ alternating them $)$ with obstacle given by the previous term $u_{n-1}$ in a domain $\Omega$ and a fixed boundary datum $g$ on $\partial \Omega$. We show that in this way we obtain an increasing sequence that converge uniformly to a viscosity solution to the minimal operator associated with $L_{1}$ and $L_{2}$, that is, the limit $u$ verifies $\min \left\{L_{1} u, L_{2} u\right\}=0$ in $\Omega$ with $u=g$ on $\partial \Omega$.
\end{abstract}

\section{INTRODUCTION}

Both the obstacle problem and maximal operators are classical subjects in the theory of PDEs and have brought the attention of many researchers for many years. For example, if one considers the family of uniformly elliptic second order operators of the form $-\operatorname{tr}\left(A D^{2} u\right)$ and look for maximal operators one finds the so-called Pucci maximal operator, $P_{\lambda, \Lambda}^{+}\left(D^{2} u\right)=\max _{A \in \mathcal{A}}-\operatorname{tr}\left(A D^{2} u\right)$, where $\mathcal{A}$ is the set of uniformly elliptic matrices with ellipticity constant between $\lambda$ and $\Lambda$, we refer to [4] for properties of these operators and details of its crucial role in regularity theory. On the other hand, the obstacle problem is a well known and widely studied free boundary problem, [10].

Our main goal in this paper is to show that one can obtain solutions to maximal or minimal operators by taking the limit of a sequence constructed iterating the obstacle problem alternating the involved operators with the previous term in the sequence as obstacle.

We will look for solutions to the Dirichlet problem for the maximum or the minimum of two operators. To this end, let $\Omega \subset \mathbb{R}^{n}$ be a bounded, smooth, domain and $g: \partial \Omega \rightarrow \mathbb{R}$ a smooth boundary condition. We want to point out that here we are not dealing with regularity issues of the solutions, therefore to simplify the presentation we set the domain and the boundary datum to be smooth.

Given an operator $L$ (notice that here we can consider fully nonlinear problems of the form $\left.L u=F\left(D^{2} u, D u, u, x\right)\right)$ we consider the obstacle problem (here solutions

Date: May 25, 2015.

2010 Mathematics Subject Classification. 35J70, 49N70, 91A15, 91A24.

Key words and phrases. Dirichlet boundary conditions, Obstacle problems, Maximal operators. 
are assumed to be above the obstacle)

$$
\left(P_{L}(\Phi, g)\right) \quad \begin{cases}u \geq \Phi & \text { in } \Omega \\ L u \geq 0 & \text { in } \Omega \\ L u=0 & \text { in }\{u>\phi\} \\ u=g & \text { on } \partial \Omega,\end{cases}
$$

or equivalently

$$
\begin{cases}\min \{L u, u-\Phi\}=0 & \text { in } \Omega \\ u=g & \text { on } \partial \Omega .\end{cases}
$$

The obstacle problem can be also stated as follows: we look for the smallest super solution of $L$ (with boundary datum $g$ ) that is above the obstacle. This formulation is quite convenient when dealing with fully nonlinear problems.

We will assume here that the problem $\left(P_{L}(\Phi, g)\right)$ has a unique viscosity solution (for general theory of viscosity solutions we refer to [4] and [5]). This is guaranteed if $L$ has a comparison principle and one can construct barriers close to the boundary so that the boundary datum $g$ is taken continuously. For general references on the obstacle problem (including regularity of solutions that are proved to be $C^{1,1}$ ) we just mention [2], [3], [6], [10] and references therein.

Now, we define a sequence of continuous functions as follows: given two continuous operators $L_{1}$ and $L_{2}$ we start with $u_{1}$ the viscosity solution to

$$
\begin{cases}L_{1} u_{1}=0 & \text { in } \Omega \\ u_{1}=g & \text { on } \partial \Omega\end{cases}
$$

and then inductively we set

$$
u_{n} \text { as the solution to } \begin{cases}P_{L_{2}}\left(u_{n-1}, g\right) & \text { for } n \text { even, } \\ P_{L_{1}}\left(u_{n-1}, g\right) & \text { for } n \text { odd. }\end{cases}
$$

That is, we define $u_{n}$ as the solution to the obstacle problem alternating the involved operator $L_{i}$ and using $u_{n-1}$ as obstacle. Note that since $u_{n-1}$ stands as the obstacle for $u_{n}$ then we trivially have $u_{n-1} \leq u_{n}$. Hence this sequence is increasing with $n$. We will also require that there exists a function $U$ that is a viscosity super solution for both operators $L_{1}$ and $L_{2}$ with boundary datum $g$ simultaneously, that is, we require that

$$
L_{1} U \geq 0, \quad L_{2} U \geq 0 \quad \text { and }\left.\quad U\right|_{\partial \Omega} \geq g .
$$

This function $U$ will be used to obtain a uniform upper bound for the increasing sequence $u_{n}$. Hypothesis (1.1) holds, for example, if the maximum principle holds for the operators or when $L_{1}$ and $L_{2}$ are proper (uniformly degenerated elliptic and non decreasing in $u$ ). In the first case we can consider $U=\max g$. While in the second one we can consider $U=c-k|x|^{2}$ where $k$ is the maximum of the ellipticity constants for $L_{1}$ and $L_{2}$ and $c$ is large enough so that $U=c-k|x|^{2} \geq g$ on $\partial \Omega$.

Note that when we consider the obstacle problem looking for sub solutions that are below the obstacle (that is, we reverse the inequalities in $\left(P_{L}(\Phi, g)\right)$ ) we can produce, iterating this obstacle problem starting with $v_{1}=u_{1}$ as above, a sequence that we call $v_{n}$. With this procedure the obtained sequence is decreasing with $n$. 
When one considers this decreasing sequence $v_{n}$ we need the existence of a function $V$ such that

$$
L_{1} V \leq 0, \quad L_{2} V \leq 0 \quad \text { and }\left.\quad V\right|_{\partial \Omega} \leq g .
$$

As before one can show that this holds if the minimum principle holds for the operators or when $L_{1}$ and $L_{2}$ are proper.

Now we are ready to state our main result that reads as follows:

\section{Theorem 1.1.}

(1) Assume that there exists $U$ such that (1.1) holds. Then the increasing sequence $u_{n}$ converges uniformly in $\bar{\Omega}$ to a viscosity solution of

$$
\begin{cases}\min \left\{L_{1} u, L_{2} u\right\}=0 & \text { in } \Omega, \\ u=g & \text { on } \partial \Omega .\end{cases}
$$

(2) Assume that there exists $V$ such that (1.2) holds. Then the decreasing sequence $v_{n}$ converges uniformly in $\bar{\Omega}$ to a viscosity solution of

$$
\begin{cases}\max \left\{L_{1} v, L_{2} v\right\}=0 & \text { in } \Omega, \\ u=g & \text { on } \partial \Omega .\end{cases}
$$

The paper is organized as follows: In Section 2 we prove Theorem 1.1 and in Section 3 we gather some remarks concerning extensions of our results.

\section{Proof of the MAIN RESUlt}

We will prove part (1) of Theorem 1.1. The proof of part (2) is entirely analogous.

Recall that we deal with viscosity solutions to the obstacle problem $\left(P_{L}(\Phi, g)\right)$ and that we assume that there is a comparison principle for the involved operators, $L_{1}$ and $L_{2}$. Let us briefly recall the definition of viscosity solution for the equation,

$$
F\left(D^{2} u, \nabla u, u, x\right)=0 .
$$

A lower semi-continuous function $u$ is a viscosity supersolution of (2.1) if for every $\phi \in C^{2}$ such that $\phi$ touches $u$ at $x \in \Omega$ strictly from below, we have

$$
F\left(D^{2} \phi, \nabla \phi, \phi, x\right) \geq 0 \text {. }
$$

An upper semi-continuous function $u$ is a subsolution of (2.1) if for every $\psi \in C^{2}$ such that $\psi$ touches $u$ at $x \in \Omega$ strictly from above, we have

$$
F\left(D^{2} \psi, \nabla \psi, \psi, x\right) \leq 0 .
$$

Finally, $u$ is a viscosity solution of (2.1) if it is both a sub- and supersolution. For general properties of viscosity solutions we refer to [5].

We assumed here that the operators $L_{1}$ and $L_{2}$ are continuous. We will comment on how to relax this hypothesis in Remark 3.4. 
Also recall that the sequence $u_{n}$ is constructed as follows: We taked $u_{1}$ the solution to

and $u_{n}$ is given inductively by

$$
\begin{cases}L_{1} u_{1}=0 & \text { in } \Omega \\ u_{1}=g & \text { on } \partial \Omega\end{cases}
$$

$$
u_{n} \text { is the solution to } \begin{cases}P_{L_{2}}\left(u_{n-1}, g\right) & \text { for } n \text { even, } \\ P_{L_{1}}\left(u_{n-1}, g\right) & \text { for } n \text { odd. }\end{cases}
$$

This sequence $u_{n}$ is increasing, bounded (since, using the comparison principle one can show by induction that $u_{n} \leq U$, where $U$ is such that (1.1) holds) and continuous, therefore by Dini's theorem there exists a continuous function $u$ such that

uniformly in $\bar{\Omega}$.

$$
u_{n} \rightarrow u
$$

Now we observe that $L_{1} u_{n} \geq 0$ if $n$ is even and $L_{2} u_{n} \geq 0$ when $n$ is odd and since $u_{2 n}$ and $u_{2 n+1}$ converge uniformly to the same limit $u$ we conclude that

$$
L_{1} u \geq 0 \quad \text { and } \quad L_{2} u \geq 0
$$

in the viscosity sense in $\Omega$.

On the other hand, we claim that

$$
\min \left\{L_{1} u_{n}, L_{2} u_{n}\right\} \leq 0
$$

for every $n$ in the viscosity sense in $\Omega$. Let us show this claim by induction. First, let us point out that it is clear that $\min \left\{L_{1} u_{1}, L_{2} u_{1}\right\} \leq 0$ since $L_{1} u_{1} \leq 0$.

Now assume that the claim holds for $u_{n}$ and let us prove it for $u_{n+1}$. In the set $\left\{u_{n+1}>u_{n}\right\}$ it holds because $L_{1} u_{n+1}$ or $L_{2} u_{n+1}$ is zero. It remains to look in the coincidence set $\left\{u_{n+1}=u_{n}\right\}$. Let $x \in\left\{u_{n+1}=u_{n}\right\} \cap \Omega$, then we have $u_{n+1}(x)=u_{n}(x)$ and $u_{n+1} \geq u_{n}$ in the whole $\Omega$. To conclude the argument we want to show that $\min \left\{L_{1} \psi(x), L_{2} \psi(x)\right\} \leq 0$ for every $\psi \in C^{2}$ that touches $u_{n+1}$ from above at $x$, but this follows since $\psi$ also touches $u_{n}$ from above at $x$.

As we have that $\min \left\{L_{1} u_{n}, L_{2} u_{n}\right\} \leq 0$ and $u_{n}$ converges uniformly to $u$ we conclude that

$$
\min \left\{L_{1} u, L_{2} u\right\} \leq 0, \quad \text { in } \Omega \text {. }
$$

As we also have $L_{1} u \geq 0$ and $L_{2} u \geq 0$ in $\Omega$ we get that

$$
\min \left\{L_{1} u, L_{2} u\right\}=0 \quad \text { in } \Omega \text {. }
$$

The boundary datum $u=g$ is taken in a pointwise sense since $u_{n}=g$ on $\partial \Omega$ and we have uniform convergence.

\section{REMARKS AND EXTENSIONS}

3.1. The maximum/minimum of two $p$-Laplacians. Recently, in [1], the authors studied the Dirichlet problem for the maximal operator associated with the $p$-Laplacian family, namely, let $\Delta_{p} u=\operatorname{div}\left(|D u|^{p-2} D u\right)$ and consider

$$
\max \left\{-\Delta_{p_{1}} u(x),-\Delta_{p_{2}} u(x)\right\}=f(x)
$$


for $2 \leq p_{1}<p_{2} \leq \infty$ in a bounded smooth domain $\Omega \subset \mathbb{R}^{N}$ with $u=g$ on $\partial \Omega$. Also the minimal operator, $\min \left\{-\Delta_{p_{1}} u(x),-\Delta_{p_{2}} u(x)\right\}=f(x)$, was considered there. They prove existence and uniqueness of a viscosity solution using PDE techniques combined with game theoretical arguments similar to the ones used in [7], [8] and [9]. Now we remark that we can use the previously described iterations with the obstacle problems for $-\Delta_{p_{1}} u$ and $-\Delta_{p_{2}} u$ to obtain a decreasing (or increasing) sequence $u_{n}$ (or $v_{n}$ ) that converges uniformly to the unique viscosity solution to the Dirichlet problem for $\max \left\{-\Delta_{p_{1}} u(x),-\Delta_{p_{2}} u(x)\right\}=f(x)$ (or for $\left.\min \left\{-\Delta_{p_{1}} u(x),-\Delta_{p_{2}} u(x)\right\}=f(x)\right)$.

3.2. Parabolic Problems. Our results can be also extended to parabolic problems. In fact we can consider the parabolic obstacle problem for a parabolic operator of the form $L(u)=F\left(u_{t}, D^{2} u, D u, u, x, t\right)$, that is,

$$
\begin{cases}u \geq \Phi & \text { in } \Omega \times(0, T), \\ L u \geq 0 & \text { in } \Omega \times(0, T), \\ L u=0 & \text { in }\{u>\Phi\} \\ u=g & \text { on } \partial \Omega \times(0, T), \\ u=u_{0} & \text { in } \Omega .\end{cases}
$$

Note that now the obstacle $\Phi$ is a function of $x$ and $t$. As before, we assume here that the problem $\left(P_{L}(\Phi, g)\right)$ has a unique viscosity solution, that the involved operators $L$ have a comparison principle and that there exists a simultaneous supersolution, $U$, valid for every $L$. In this way we obtain an increasing and bounded sequence that converge to a viscosity solution to

$$
\begin{cases}\min \left\{L_{1} u(x, t), L_{2} u(x, t)\right\}=0 & \text { in } \Omega \times(0, T) \\ u=g & \text { on } \partial \Omega \times(0, T) \\ u=u_{0} & \text { in } \Omega .\end{cases}
$$

Combining previous remarks we obtain existence of a viscosity solution to

$$
\begin{cases}\min \left\{u_{t}-\Delta_{p_{1}} u, u_{t}-\Delta_{p_{1}} u\right\}(x, t)=0 & \text { in } \Omega \times(0, T), \\ u=g & \text { on } \partial \Omega \times(0, T), \\ u=u_{0} & \text { in } \Omega .\end{cases}
$$

3.3. Maximum/minimum of more than two operators. Let us mention that this idea works for a finite number of operators, $L_{1}, \ldots, L_{K}$. We just have to consider the obstacle problem for $L_{1}, \ldots, L_{K}$ and iterate to produce an increasing (or decreasing) sequence that converges uniformly to a viscosity solution to $\max _{j}\left\{L_{j} u(x)\right\}=0$ (or to $\min _{j}\left\{L_{j} u(x)\right\}=0$ ).

This procedure can be also extended to a sequence of operators $\left\{L_{j}\right\}_{j \in \mathbb{N}}$ the only point is that the obstacle problem for every operator in the sequence has to appear infinitely many times (this can be done just by considering the sequence $\left.P_{L_{1}}, P_{L_{2}}, P_{L_{1}}, P_{L_{2}}, P_{L_{3}}, P_{L_{1}}, \ldots.\right)$.

We can also consider an arbitrary family of operators $\left\{L_{i}\right\}_{i \in I}$ (here the set of indexes $I$ is not assumed to be numerable). To this end we have to modify slightly our previous procedure. Before proceeding with this extension we have to recall 
some definitions for two reasons: first, to complete the technical details omitted in the proof of Theorem 1.1 related to the fact that we can consider non continuous operators (as we do in Remark 3.4) and second because, even when all the operators $\left\{L_{i}\right\}_{i \in I}$ are continuous, the $\inf _{i \in I}\left\{L_{i}\right\}$ it is not necessarily so.

Given $F: S(n) \times \mathbb{R}^{n} \times \mathbb{R} \times \Omega \rightarrow \mathbb{R}$ where $S(n)$ denotes the set of symmetric $n \times n$ matrices. We consider the lower semicontinous (denoted as $F_{*}$ ) and upper semicontinous (called $F^{*}$ ) envelopes of $F$. These functions are given by

$$
\left\{\begin{array}{l}
F^{*}(M, v, r, x)=\limsup _{(N, w, s, y) \rightarrow(M, v, r, x)} F(N, w, s, y), \\
F_{*}(M, v, r, x)=\underset{(N, w, s, y) \rightarrow(M, v, r, x)}{\liminf } F(N, w, s, y) .
\end{array}\right.
$$

These functions coincide with $F$ at every point of continuity of $F$ and are lower and upper semicontinous respectively. With these envelopes at hand, we consider viscosity solutions for the equation,

$$
F\left(D^{2} u, \nabla u, u, x\right)=0,
$$

when $F$ is not necessary continuous. In this case when dealing with super solutions of (3.1) we impose that

$$
F^{*}\left(D^{2} \phi, \nabla \phi, \phi, x\right) \geq 0,
$$

for every $\phi \in C^{2}$ that touches $u$ at $x \in \Omega$ strictly from below; while for a sub solution of (3.1) we ask for

$$
F_{*}\left(D^{2} \psi, \nabla \psi, \psi, x\right) \leq 0,
$$

when $\psi \in C^{2}$ touches $u$ at $x \in \Omega$ strictly from above. Finally, $u$ is a viscosity solution of (3.1) if it is both a sub- and super solution.

Now, let us construct our sequence. We start by solving the Dirichlet problem for all the operators $L_{i}$, that is, we let $u_{1}^{i}$ be such that

$$
\begin{cases}L_{i} u_{1}^{i}=0 & \text { in } \Omega, \\ u_{1}^{i}=g & \text { on } \partial \Omega,\end{cases}
$$

and then take

$$
u_{1}=\sup _{i \in I} u_{1}^{i}
$$

Now we define inductively $u_{n}$ by taking the supremum of the solutions to the obstacle problem for the operators $L_{i}$ with obstacle $u_{n-1}$, that is, we take $u_{n}^{i}$ the solution to

and then we let

$$
\begin{cases}u_{n}^{i} \geq u_{n-1} & \text { in } \Omega \\ L_{i} u_{n}^{i} \geq 0 & \text { in } \Omega \\ L_{i} u_{n}^{i}=0 & \text { in }\left\{u_{n}^{i}>u_{n-1}\right\} \\ u_{n}^{i}=g & \text { on } \partial \Omega\end{cases}
$$

$$
u_{n}=\sup _{i \in I} u_{n}^{i}
$$

As was argued previously, assuming that there is an upper bound for our sequence, $U$, we have that there exists $u$ such that

$$
u_{n} \rightarrow u
$$


uniformly in $\bar{\Omega}$.

Now our aim is to show that $u$ is a viscosity solution to

$$
L u=\inf _{i \in I} L_{i} u=0 .
$$

First, we observe that, given $i \in I$, we have $u_{n}^{i} \rightarrow u$ because $u_{n} \leq u_{n}^{i} \leq u_{n+1}$. As for each $i \in I$ we know that $L_{i} u_{n}^{i} \geq 0$ in $\Omega$, we get that $u$ is a supersolution of $L_{i} u=0$, this is $L_{i} u \geq 0$. Hence it is a supersolution of (3.2), in the sense that $L u \geq 0$ in the viscosity sense.

Let us now show that $u_{n}$ is a subsolution of (3.2) for all $n \in \mathbb{N}$. We proceed by induction. For $n=1$, we consider an arbitrary $x_{0} \in \Omega$. Let $\psi$ be an arbitrary smooth function that touches from above $u_{1}$ at $x_{0}$, then there exist $\left\{i_{k}\right\}_{k \in \mathbb{N}} \subset I$ such that $u_{1}\left(x_{0}\right)=\lim _{k} u_{1}^{i_{k}}\left(x_{0}\right)$. Then let $x_{k}$ be a point where $\psi-u_{1}^{i_{k}}$ attains its minimum, we know that $x_{k} \rightarrow x_{0}$. We have that $\psi-\psi\left(x_{k}\right)+u_{1}^{i_{k}}\left(x_{k}\right)$ touches from above $u_{1}^{i_{k}}$ at $x_{k}$. Then, since $L_{i_{k}} u_{1}^{i_{k}}=0$, we get

$$
L_{i_{k}}\left(D^{2} \psi\left(x_{k}\right), \nabla \psi\left(x_{k}\right), u_{1}^{i_{k}}\left(x_{k}\right), x_{k}\right) \leq 0 .
$$

Hence

$$
L\left(D^{2} \psi\left(x_{k}\right), \nabla \psi\left(x_{k}\right), u_{1}^{i_{k}}\left(x_{k}\right), x_{k}\right) \leq 0
$$

and we can conclude that

$$
L_{*} \psi\left(x_{0}\right) \leq \lim _{k} L\left(D^{2} \psi\left(x_{k}\right), \nabla \psi\left(x_{k}\right), \psi-\psi\left(x_{k}\right)+u_{1}^{i_{k}}\left(x_{k}\right), x_{k}\right) \leq 0
$$

We have proved that $u_{1}$ is a viscosity subsolution of (3.2).

Analogously, we can show that the claim holds for $u_{n+1}$ assuming that it holds for $u_{n}$. We consider an arbitrary $x_{0} \in \Omega$. Let $\psi$ be an arbitrary smooth function that touches from above $u_{n+1}$ at $x_{0}$, then, as before, there exist $\left\{i_{k}\right\}_{k \in \mathbb{N}} \subset I$ such that $u_{n+1}\left(x_{0}\right)=\lim _{k} u_{n+1}^{i_{k}}\left(x_{0}\right)$. Then let $x_{k}$ be a point where $\phi-u_{n+1}^{i_{k}}$ attains its minimum, we know that $x_{k} \rightarrow x_{0}$. We have that $\psi-\psi\left(x_{k}\right)+u_{n+1}^{i_{k}}\left(x_{k}\right)$ touches from above $u_{n+1}^{i_{k}}$ at $x_{k}$. Then, since $L_{i_{k}} u_{n+1}^{i_{k}}=0$ in $\left\{u_{n+1}^{i_{k}}>u_{n}\right\}$ and $L_{*} u_{n+1}^{i_{k}} \leq 0$ in $\left\{u_{n+1}^{i_{k}}=u_{n}\right\}$ by the inductive hypothesis, we get

$$
L_{*} u_{n+1}^{i_{k}} \leq 0 .
$$

Hence

$$
L_{*}\left(D^{2} \psi\left(x_{k}\right), \nabla \psi\left(x_{k}\right), u_{1}^{i_{k}}\left(x_{k}\right), x_{k}\right) \leq 0
$$

and we can conclude that

$$
L_{*} \psi\left(x_{0}\right) \leq \lim _{k} L_{*}\left(D^{2} \psi\left(x_{k}\right), \nabla \psi\left(x_{k}\right), u_{1}^{i_{k}}\left(x_{k}\right), x_{k}\right) \leq 0
$$

We have proved that $u_{n+1}$ is a subsolution of (3.2), this is $L_{*} u_{n+1} \leq 0$.

Finally, being the limit of subsolutions, we conclude that $u$ (the limit of the sequence $u_{n}$ ) is a subsolution and therefore a solution of (3.2). 
3.4. On the continuity hypothesis. In the proof of the main theorem and Remark 3.3 we assume that the operators were continuous. We used this fact in two steps.

We conclude that $u$ was a supersolution of $L_{i} u=0$ (as being the limit of supersolutions) and then, because of this, that it was a super solution of $\inf _{i \in I} L_{i} u=0$. But this fact is not necessarily true when the operators are not continuous. We have that $L_{i}^{*} u \geq 0$ and hence we can conclude that $\inf _{i \in I} L_{i}^{*} u \geq 0$ but we want to conclude that $\left(\inf _{i \in I} L_{i}\right)^{*} u \geq 0$. So we need to require that $\left(\inf _{i \in I} L_{i}\right)^{*} \geq \inf _{i \in I} L_{i}^{*}$, that holds when the operator are continuous.

On the other hand we need that $\left(\inf _{i \in I} L_{i}\right)_{*} \leq \inf _{i \in I} L_{i *}$. In the proof of the main theorem we need this fact to conclude that $u_{n+1}$ is a subsolution of $\min \left\{L_{1}, L_{2}\right\} u=$ 0 on the set $\left\{u_{n+1}>u_{n}\right\}$ where we know $L_{1} u_{n+1}$ or $L_{2} u_{n+1}$ is zero. In Remark 3.3 we need it in a similar way when we conclude that $L_{*} u_{n+1}^{i_{k}} \leq 0$. In this case we have that this inequality always holds.

In conclusion, when we have that the involved operators are not continuous we need to require that

$$
\left(\inf _{i \in I} L_{i}\right)^{*} \geq \inf _{i \in I} L_{i}^{*}
$$

Let us present two simple examples where this assumption does not hold. We consider $\Omega=(0,1)$ and the boundary datum $g(0)=g(1)=0$ in both examples.

Example 1. We consider $L_{1}=-u^{\prime \prime}-\chi_{[0,1 / 2)}$ and $L_{2}=-u^{\prime \prime}-\chi_{[1 / 2,1]}$, then

$$
\left(\inf \left\{L_{1}, L_{2}\right\}\right)^{*}=\left(-u^{\prime \prime}-\chi_{[0,1]}\right)^{*}=-u^{\prime \prime}-\chi_{[0,1]}
$$

while

$$
\inf \left\{L_{1}^{*}, L_{2}^{*}\right\}=\inf \left\{-u^{\prime \prime}-\chi_{[0,1 / 2)},-u^{\prime \prime}-\chi_{[1 / 2,1]}\right\}=-u^{\prime \prime}-\chi_{[0,1 / 2) \cup(1 / 2,1]} .
$$

Hence, $\left(\inf _{i \in I} L_{i}\right)^{*} \geq \inf _{i \in I} L_{i}^{*}$ does not hold pointwise. However, remark that in this example we have the same solutions for $\inf _{i \in I} L_{i}^{*} u=0$ and for $\inf _{i \in I} L_{i} u=0$.

Example 2. Now we consider $L_{i}=-u^{\prime \prime}-\delta_{i}$ for $i \in[0,1]$ (remark that in this example we have an uncountable family of operators), then

$$
\left(\inf _{i \in I} L_{i}\right)^{*}=\left(-u^{\prime \prime}-\chi_{[0,1]}\right)^{*}=-u^{\prime \prime}-\chi_{[0,1]}
$$

while

$$
\inf _{i \in I} L_{i}^{*}=\inf _{i \in I}-u^{\prime \prime}=-u^{\prime \prime}
$$

Again in this example the hypothesis $\left(\inf _{i \in I} L_{i}\right)^{*} \geq \inf _{i \in I} L_{i}^{*}$ does not hold pointwise. Note that now the equations $\inf _{i \in I} L_{i}^{*} u=0$ and $\inf _{i \in I} L_{i} u=0$ are really different.

\section{REFERENCES}

[1] P. Blanc, J. P. Pinasco and J. D. Rossi. Maximal operators for the p-Laplacian family. Preprint.

[2] L. A. Caffarelli. The regularity of free boundaries in higher dimensions. Acta Math., 139(3-4) (1977), 155-184.

[3] L. A. Caffarelli. The obstacle problem revisited. J. Fourier Anal. Appl., 4(4-5), (1998), 383402. 
[4] L. A. Caffarelli and X. Cabre, Fully nonlinear elliptic equations. Amer. Math. Soc. Colloquium Publications, 43. Providence, RI, 1995.

[5] M. G. Crandall, H. Ishii and P. L. Lions. User's guide to viscosity solutions of second order partial differential equations. Bull. Amer. Math. Soc., 27, (1992), 1-67.

[6] P. Daskalopoulos and P. M. N. Feehan. $C^{1,1}$ Regularity for Degenerate Elliptic Obstacle Problems in Mathematical Finance. Preprint.

[7] J. J. Manfredi, M. Parviainen and J. D. Rossi. On the definition and properties of $p$ harmonious functions. Ann. Scuola Nor. Sup. Pisa, 11, (2012), 215-241.

[8] Y. Peres, O. Schramm, S. Sheffield and D. Wilson, Tug-of-war and the infinity Laplacian. J. Amer. Math. Soc., 22, (2009), 167-210.

[9] Y. Peres, S. Sheffield, Tug-of-war with noise: a game theoretic view of the p-Laplacian, Duke Math. J., 145(1), (2008), 91-120.

[10] A. Petrosyan, H. Shagholian, and N. Uraltseva, Regularity of free boundaries in obstacle type problems, American Mathematical Society, Providence, RI, 2012.

Depto. Matemática, FCEyn, Buenos Aires University,

Ciudad Universitaria, Pab 1 (1428),

Buenos Aires, Argentina.

pblanc@dm.uba.ar, jpinasco@dm.uba.ar, jrossi@dm.uba.ar 